Präv Gesundheitsf 2014 · 9:67-68

DOI 10.1007/s11553-014-0433-6

Online publiziert: 1. April 2014

(c) Springer-Verlag Berlin Heidelberg 2014

\title{
Mathilde Kersting
}

Forschungsinstitut für Kinderernährung, Institut an der Rheinischen Friedrich-Wilhelms-Universität Bonn, Dortmund, Deutschland

\section{Editorial}

von Demenz gefördert wird, aber zur Erreichung inaktiver Personen mit niedrigem Bildungsstand proaktive Rekrutierungswege angezeigt sind.

\section{Im Bereich Familie/ Kinder/Schule:}

- dass potentielle Missverständnisse bei den zentralen Begriffen der Säuglingsernährung identifiziert werden müssen, damit auch Familien mit Migrationshintergrund an den hiesigen Präventionsangeboten partizipieren können;

- dass Eltern mehr als bisher in präventive Programme im Kindes- und Jugendbereich eingebunden werden sollten, um riskanten Verhaltensweisen bei Kindern und Jugendlichen im Umgang mit Alkohol vorzubeugen;

- dass eine Umstellung vom Schulweg mit dem Auto zum zu Fuß zur Schule gehen als sektorübergreifende Strategie die Gesundheit der Kinder nicht nur durch vermehrte Bewegung sondern zusätzlich durch eine Reduktion des $\mathrm{CO}_{2}$-Ausstoßes fördert.

\section{Im Bereich Arbeitswelt:} eine verstärkte Interdisziplinarität der gesundheitlichen Versorgung nahe liegt;

- dass Angebote der Bewegungsförderung für Ältere im Lebensumfeld Kommune mehr als nur die ursprüngliche Zielgruppe erreichen, wenn in Mehrkomponenten-Interventionen auch der Bekanntheitsgrad der Angebote gesteigert wird;

- dass durch Integration von Bewegung in den Alltag die kognitive Leistungsfähigkeit im Sinne der Prävention
- dass eine hohe Stressbelastung und beeinträchtigte Lebenszufriedenheit im Berufsalltag weniger durch charakteristische Aufgaben z. B. der Berufsfeuerwehr im Außendienst, sondern durch alltäglichen Innendienst entstehen;

- dass Beschäftigte mit einem möglichen Zielkonflikt zwischen dem Risiko, bei ansteckender Krankheit zur Arbeit zu gehen und der maximalen
Einsatznotwendigkeit bei einer Pandemie souverän umgehen können;

- dass auf Erzieher in dem neuen Aufgabenfeld der Krippenbetreuung von 0 - bis 3-Jährigen auch neue physische und psychische Herausforderungen bis hin zur stimmlichen Beanspruchung zukommen;

- dass es sich auf dem Weg zu einer „praxisbasierten Evidenz" für die Planung und Umsetzung von Projekten der betrieblichen Gesundheitsförderung empfiehlt, Erfahrungen und konkrete Handlungsanleitungen aus anderen Projekten einzubeziehen;

- dass fundierte Nachweise über die Wirksamkeit betrieblicher Gesundheitsförderung auf Stressreduktion, Wohlbefinden und klinisch bedeutsame Vorteile für stressbelastete Beschäftigte nach wie vor fehlen.

\section{Auf internationaler Ebene:}

- dass Forschung im Gebiet des „Public Health“ als Grundlage für eine zielgerichtete, internationale und interdisziplinäre Maßnahmenentwicklung im Kampf gegen den Menschenhandel gefragt ist.

Zusammengenommen besteht eine große Herausforderung der Prävention und Gesundheitsförderung nach wie vor darin, niederschwellige und wirksame Wege zu schwer zugänglichen Bevölkerungsgruppen zu finden, bei denen gleichzeitig gesundheitliche Risiken akkumulieren. Dabei ist selbstverständlich eine ,Evidenzbasierung' der Maßnahmen anzustreben, aber auf dem Wege dorthin sind Empirie und plausibler Pragmatismus weiterhin gefragt. Jeder Schritt einer punktu- 


\section{Editorial}

ellen wissenschaftlichen Absicherung ist ein Fortschritt im „Public Health“.

Das Forschungsinstitut für Kinderernährung hat dies an einem kleinen Ausschnitt der frühen Prävention, der gesunden Säuglingsernährung, erläutert (Hilbig A, Lentze MJ, Kersting M. Monatsschr Kinderheilkd 2012; 160:1089-1095).

In diesem Sinne lohnt es sich, gelegentlich einen Blick über den eigenen ,Tellerrand' zu werfen; die Artikel in diesem Heft bieten hierzu gute Gelegenheiten.

9. Whily

\section{Kersting}

\section{Korrespondenzadresse}

Prof. Dr. M. Kersting
Forschungsinstitut für
Kinderernährung
Institut an der
Rheinischen Friedrich-
Wilhelms-Universität Bonn
Heinstück 11
44225 Dortmund
kersting@fke-do.de

